\title{
Novel ultra-high-frequency electrocardiogram tool for the description of the ventricular depolarization pattern before and during cardiac resynchronization
}

Citation for published version (APA):

Jurak, P., Curila, K., Leinveber, P., Prinzen, F. W., Viscor, I., Plesinger, F., Smisek, R., Prochazkova, R., Osmancik, P., \& Halamek, J. (2020). Novel ultra-high-frequency electrocardiogram tool for the description of the ventricular depolarization pattern before and during cardiac resynchronization. Journal of Cardiovascular Electrophysiology, 31(1), 300-307. https://doi.org/10.1111/jce.14299

Document status and date:

Published: 01/01/2020

DOI:

10.1111/jce.14299

Document Version:

Publisher's PDF, also known as Version of record

Document license:

Taverne

Please check the document version of this publication:

- A submitted manuscript is the version of the article upon submission and before peer-review. There can be important differences between the submitted version and the official published version of record.

People interested in the research are advised to contact the author for the final version of the publication, or visit the DOI to the publisher's website.

- The final author version and the galley proof are versions of the publication after peer review.

- The final published version features the final layout of the paper including the volume, issue and page numbers.

Link to publication

\footnotetext{
General rights rights.

- You may freely distribute the URL identifying the publication in the public portal. please follow below link for the End User Agreement:

www.umlib.nl/taverne-license

Take down policy

If you believe that this document breaches copyright please contact us at:

repository@maastrichtuniversity.nl

providing details and we will investigate your claim.
}

Copyright and moral rights for the publications made accessible in the public portal are retained by the authors and/or other copyright owners and it is a condition of accessing publications that users recognise and abide by the legal requirements associated with these

- Users may download and print one copy of any publication from the public portal for the purpose of private study or research.

- You may not further distribute the material or use it for any profit-making activity or commercial gain

If the publication is distributed under the terms of Article 25fa of the Dutch Copyright Act, indicated by the "Taverne" license above, 


\title{
Novel ultra-high-frequency electrocardiogram tool for the description of the ventricular depolarization pattern before and during cardiac resynchronization
}

\author{
Pavel Jurak $\mathrm{PhD}^{1}{ }^{1}$ | Karol Curila MD, $\mathrm{PhD}^{2}$ ( $\mid$ Pavel Leinveber $\mathrm{MS}^{3,4} \mid$ \\ Frits W. Prinzen PhD $^{5}$ | Ivo Viscor PhD ${ }^{1}$ | Filip Plesinger PhD ${ }^{1}$ | Radovan Smisek MS $^{1,6}$ | \\ Radka Prochazkova MS ${ }^{2}$ | Pavel Osmancik MD, PhD ${ }^{2}$ | Josef Halamek PhD ${ }^{1}$ (1) | \\ Magdalena Matejkova $^{3}$ | Jolana Lipoldova ${ }^{3,4}$ | Miroslav Novak ${ }^{3,4}$ | Roman Panovsky,4 | \\ Petr Andrla $^{1}$ | Vlastimil Vondra ${ }^{1}$ | Petr Stros ${ }^{2}$ | Jana Vesela ${ }^{2}$ | Dalibor Herman ${ }^{2}$
}

${ }^{1}$ Institute of Scientific Instruments, The Czech Academy of Sciences, Brno, Czech Republic

${ }^{2}$ Department of Cardiology, Cardiocenter, Third Faculty of Medicine, Charles University, University Hospital Kralovske Vinohrady, Prague, Czech Republic

${ }^{3}$ International Clinical Research Center, St Anne's University Hospital, Brno,

Czech Republic

${ }^{4}$ First Department of Internal MedicineCardioangiology, Faculty of Medicine of Masaryk University, St Anne's University Hospital, Brno, Czech Republic

${ }^{5}$ Department of Physiology, Cardiovascular Research Institute Maastricht, Maastricht University, Maastricht, The Netherlands

${ }^{6}$ Department of Biomedical Engineering, The Faculty of Electrical Engineering and Communication, Brno University of Technology, Brno, Czech Republic

\section{Correspondence}

Pavel Jurak, PhD, Institute of Scientific Instruments, The Czech Academy of Sciences, Kralovopolska 147, 63500 Brno, Czech Republic.

Email: jurak@isibrno.cz

Disclosures: Frits W. Prinzen received research grants from Medtronic, Abbott, Biotronik, Microport, and Biosense Webster. The other authors report no conflicts of interest.

Funding information

Grantová Agentura Ceské Republiky, Grant/Award Number: GA17-13830S;

Ministry of Education, Youth, and Sports of the Czech Republic, Grant/Award Numbers: LO1212, LQ1605; Charles University Research Program Q38 and Research Centre program, Grant/Award Number: UNCE/MED/002

\begin{abstract}
Introduction: The present study introduces a new ultra-high-frequency 14-lead electrocardiogram technique (UHF-ECG) for mapping ventricular depolarization patterns and calculation of novel dyssynchrony parameters that may improve the selection of patients and application of cardiac resynchronization therapy (CRT).

Methods: Components of the ECG in sixteen frequency bands within the 150 to $1000 \mathrm{~Hz}$ range were used to create ventricular depolarization maps. The maximum time difference between the UHF QRS complex centers of mass of leads V1 to V8 was defined as ventricular electrical dyssynchrony (e-DYS), and the duration at $50 \%$ of peak voltage amplitude in each lead was defined as the duration of local depolarization (Vd). Proof of principle measurements was performed in seven patients with left (left bundle branch block) and four patients with right bundle branch block (right bundle branch block) before and during CRT using biventricular and His-bundle pacing.

Results: The acquired activation maps reflect the activation sequence under the tested conditions. e-DYS decreased considerably more than QRS duration, during both biventricular pacing $(-50 \%$ vs $-8 \%)$ and His-bundle pacing $(-77 \%$ vs $-13 \%)$. While biventricular pacing slightly increased $\mathrm{Vd}$, His-bundle pacing reduced $\mathrm{Vd}$ significantly $(+11 \%$ vs $-36 \%)$, indicating the contribution of the fast conduction system. Optimization of biventricular pacing by adjusting VV-interval showed a decrease of e-DYS from 102 to 36 ms with only a small Vd increase and QRS duration decrease.

Conclusions: The UHF-ECG technique provides novel information about electrical activation of the ventricles from a standard ECG electrode setup, potentially improving the selection of patients for CRT and application of CRT.
\end{abstract}

KEYWORDS

biventricular pacing, cardiac resynchronization therapy, His-bundle pacing, ultra-high-frequency ECG, ventricular electrical dyssynchrony 


\section{1 | INTRODUCTION}

Cardiac resynchronization therapy (CRT) represents an effective technique for the treatment of patients with heart failure having ventricular dyssynchrony. However, $30 \%$ to $50 \%$ of CRT recipients do not have a clear positive echocardiographic and/or clinical outcome. ${ }^{1,2}$ This has been attributed to imperfect patient selection, incorrectly implemented CRT technology, and suboptimal device programming.

The primary goal of resynchronization is to eliminate electromechanical dyssynchrony. QRS width and morphology reflect the presence of ventricular dyssynchrony to some extent, but in principle, it provides indirect measures. The concept presented here uses ultra-high-frequency electrocardiogram (ECG) to quantify conduction and electrical dyssynchrony (e-DYS) in the ventricles.

High-frequency $(150-300 \mathrm{~Hz})$ 12-lead ECG (HF-ECG) has been recognized more than half a century ago. With by then limited technologies, ischemia, and infarction were localized by using HF components $^{3,4}$ that are spatially variable and thus lead dependent. ${ }^{5}$ Recently, our group has started to apply HF-ECG to determine e-DYS. MADIT-CRT database ${ }^{6}$ study introduced a ventricular electrical delay (VED) determined in 676 left bundle branch block (LBBB) patients. ${ }^{7}$ The results showed that VED predicts survival in biventricular resynchronization (BiV) patients better than the conventional QRS-derived parameters. A more recent study indicated that VED might be a more useful predictor for CRT response than ECG characteristics of strict LBBB. ${ }^{8}$ Simultaneously, the concept started with a single-band ultra-highfrequency $(500-1000 \mathrm{~Hz}) 12$-lead ECG ${ }^{9}$ presented measures of electrical depolarization patterns, among others quantifying the effect of BiV.

This study aims to introduce a new methodology based on broadband ultra-high-frequency $(150-1000 \mathrm{~Hz})$ 14-lead ECG technique (UHF-ECG), extending the above-mentioned approaches to more robust and comprehensive imaging of electrical activation patterns and quantification of e-DYS and local depolarization duration. The main advantage of the new approach is the possibility of using UHFECG directly during the implantation of the pacemaker.

\section{2 | METHODS}

\section{1 | Data acquisition}

Measurements were performed at the International Clinical Research Center at St Anne's University Hospital, Brno, Czech Republic and Cardiocenter of Faculty Hospital Kralovske Vinohrady and the Third Medical Faculty of Charles University, Prague, Czech Republic. Seven patients with left (LBBB) and four patients with right bundle branch block (RBBB) before and during CRT using biventricular and His-bundle pacing were selected for publication, Table 1. All subjects gave their informed consent to the investigation. The Ethics Committee of St Anne's University Hospital, Brno and Faculty Hospital Kralovske Vinohrady, Prague approved the study.

A VDI monitor (Ventricular Dyssynchrony Imaging monitor; ISI, Cardion, FNUSA, CZ, 2018) was used to record and analyze $5 \mathrm{kHz}$ ECG signals with a dynamic range of 26 bits ( $3 \mathrm{nV}$ resolution) and a frequency range of $1.5 \mathrm{kHz}$. UHF-ECG data were collected over 1 to 5 minutes in a resting supine position with a standard 14-lead ECG setup (Figure $1 \mathrm{~A}$ ). All presented methods and results can be related to a 12-lead ECG by omitting precordial leads V7 and V8.

\section{2 | Data preprocessing}

A digital $50 \mathrm{~Hz}$ notch filter was used for the elimination of power-line interference. In ECG acquired during CRT ON the pacing, artifacts were eliminated as described in. ${ }^{10}$

Subsequently, QRS complexes were detected ${ }^{11}$ and clustered into different QRS morphology categories using a robust multichannel

TABLE 1 Patient baseline characteristics

\begin{tabular}{|c|c|c|c|c|c|c|c|c|c|}
\hline Patient & Age & Gender & Diagnosis & CRT & AA medication & $\begin{array}{l}\text { Conduction } \\
\text { disturbances }\end{array}$ & LVEF \% & NYHA & specification \\
\hline 1 & 76 & $M$ & DCM & Biv & Amiodarone & LBBB & 18 & III & Mitral regurgitation IV. \\
\hline 2 & 78 & $M$ & IHD & Biv & Bisoprolol & LBBB & 35 & II & Scar \\
\hline 3 & 74 & $\mathrm{~F}$ & IHD & Biv & Metoprolol, amiodarone & LBBB & 28 & III & Scar, artificial mitral valve \\
\hline 4 & 69 & $M$ & DCM & Biv & Betaloc, procoralan & LBBB & 19 & II-III & $\begin{array}{l}\text { Mitral and tricuspid valve } \\
\text { regurgitation }\end{array}$ \\
\hline 5 & 74 & $M$ & $\mathrm{DCM}+\mathrm{IHD}$ & Biv & Bisoprolol & $\mathrm{RBBB}+\mathrm{LAH}$ & 15 & III & \\
\hline 6 & 68 & $M$ & IHD & Biv & 0 & $\mathrm{RBBB}+\mathrm{LAH}$ & 25 & III & \\
\hline 7 & 72 & $M$ & AV block & His & 0 & RBBB & 55 & II & \\
\hline 8 & 88 & $\mathrm{~F}$ & SSSy & His & 0 & $\mathrm{RBBB}+\mathrm{LAH}$ & 55 & II & Artificial Ao valve \\
\hline 9 & 80 & $M$ & IHD & His & metoprolol & LBBB & 33 & III & \\
\hline 10 & 90 & $\mathrm{~F}$ & IHD & His & 0 & LBBB & 38 & III & \\
\hline 11 & 91 & $M$ & DCM & His & bisoprolol & LBBB & 30 & II & \\
\hline
\end{tabular}

Abbreviations: DCM, dilated cardiomyopathy; IHD, ischemic heart disease; LAH, left anterior hemiblock; LBBB, left bundle branch block; LVEF, left ventricular ejection fraction; M/F, male/female; NYHA, New York Heart Association Classification; RBBB, right bundle branch block; SSSy, sick sinus syndrome; VHD, valvular heart disease. 
(A)

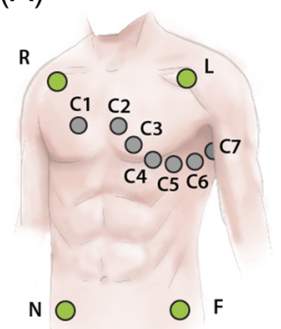

$\mathrm{NO}$ $\bigcirc$
Single examination

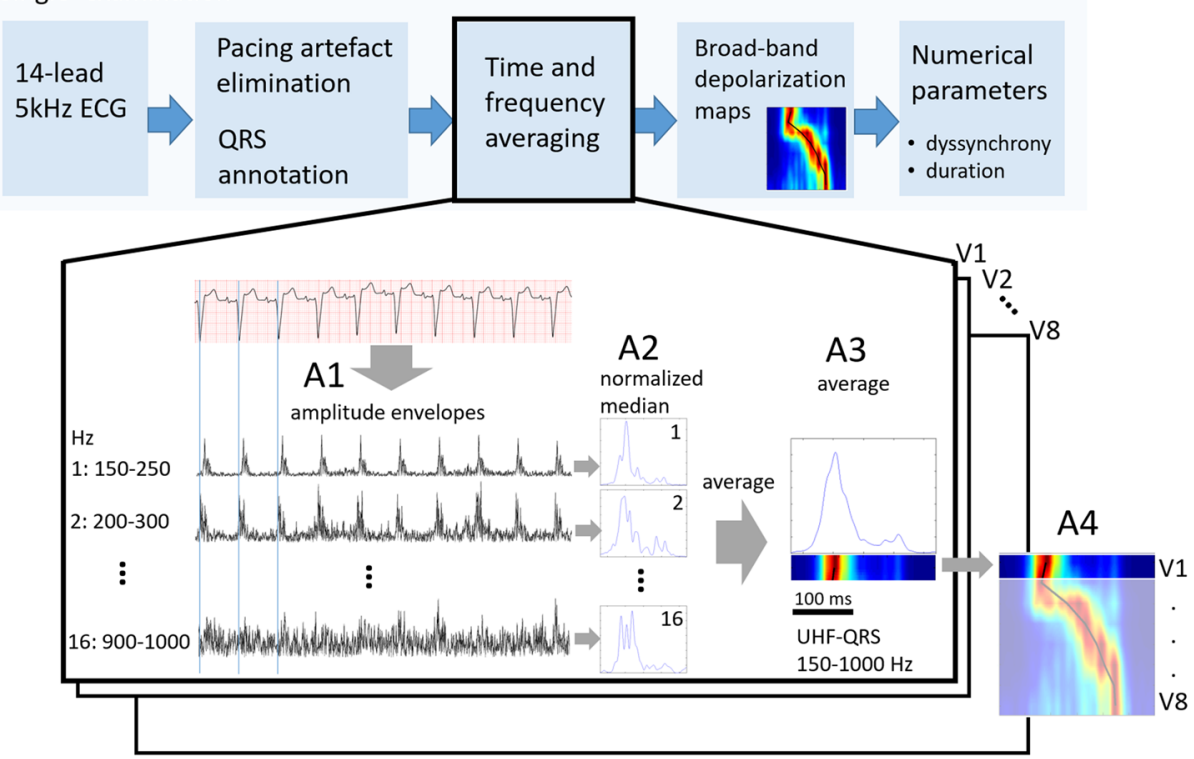

(B)

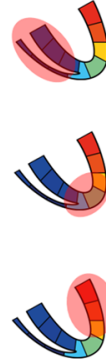

ventricular depolarization map

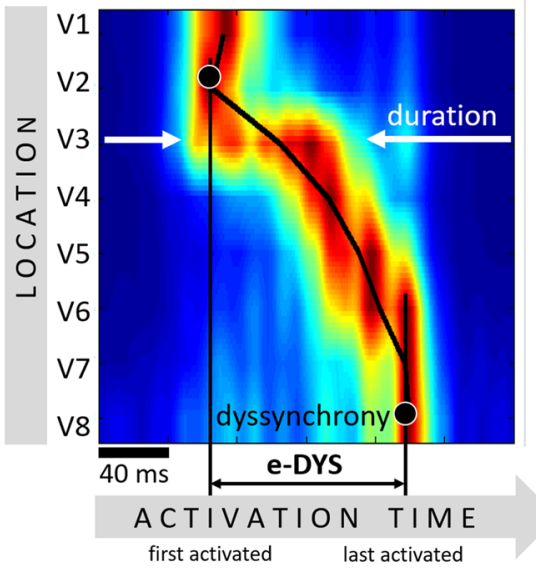

activation duration graph

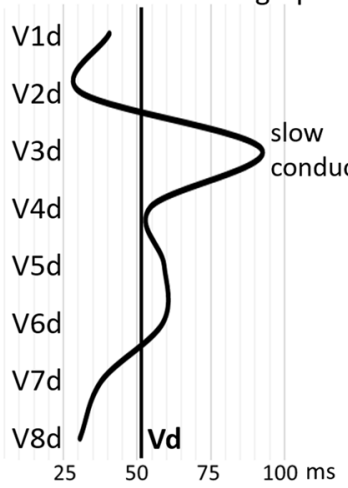

center of mass - local activation time
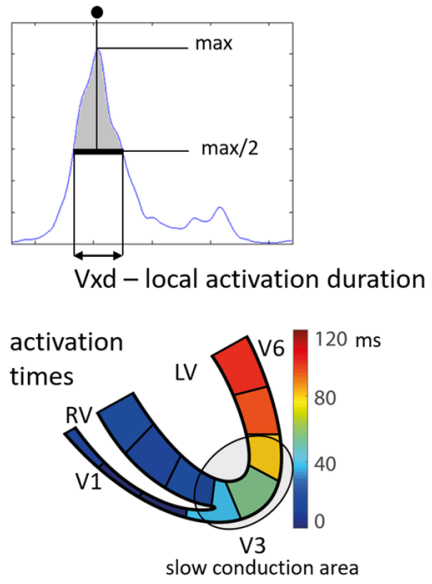

color intensity corresponds to the time distribution of simultaneously depolarized myocardial cells

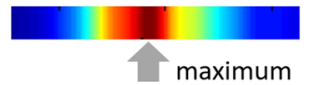

FIGURE 1 UHF-ECG computational procedure. Patient 1, Left bundle branch block (LBBB). A, Schematic interpretation of UHF-ECG computational steps. A1-amplitude envelopes computation in 16 frequency bands, A2-the normalized amplitude median in the single frequency band, A3-average amplitude shape overall frequency bands (UHF-QRS). A4-color interpretation of UHF-QRSs. Each horizontal row of the map represents the normalized shape of UHF-QRS for one $\mathrm{V}$ lead, the maximum amplitude in each row is represented in the dark red and the minimum in the dark blue. B, Ventricular depolarization map. The colors indicate time (horizontal axis) and spatial (vertical axis) depolarization distribution. e-DYS is determined as the distance between the first (here lead V2) and the last (here lead V8) local activation times. The local activation duration $(\mathrm{Vxd})$ is determined as the duration of depolarization activity in single lead in $50 \%$ of the maximum. High $\mathrm{Vxd}$ values point to an area of slow conduction. Vd represents mean from all Vxds. e-DYS, electrical dyssynchrony; UHF-ECG, ultrahigh-frequency 14-lead ECG technique

approach capable of recognizing sinus QRS patterns as well as paced and irregular patterns. In this study, the sinus and paced rhythm were used with the elimination of extra-systoles.

\section{3 | Broad-band ventricular depolarization maps}

Averaging in time and frequency domain was used to achieve a high signal to noise ratio of the weak UHF components. For each precordial lead (V1-V8), the amplitude envelopes were computed in 16 frequency bands 150 to 250,200 to 300,250 to $350, \ldots 850$ to 950, and 900 to $1000 \mathrm{~Hz}$ using the Hilbert transform (Figure 1A, A1). Amplitude envelopes were segmented by QRS annotation. In each frequency band of each ECG channel, the median amplitude envelopes were computed as the median value of the points of the segmented envelopes during the QRS complex. The median amplitude envelopes of each frequency band were then normalized 
for each frequency band. Normalization was performed using the integral, which was calculated in the interval of $120 \mathrm{~ms}$ before the annotation and $120 \mathrm{~ms}$ after the annotation. Subsequently, all median envelope values were divided by this integral. This procedure achieves that the integral in all frequency bands is the same (Figure 1A, A2). Normalization in the various frequency domains was used to avoid that the larger low-frequency amplitudes would dominate the weak high-frequency amplitudes during the subsequent averaging over frequencies.

The broad-band QRS complex (UHF-QRS) was then constructed as the average of the 16 normalized median amplitude envelopes (Figure 1A, A3). UHF-QRS complexes from all precordial leads can be displayed as a color map (Figure 1A, A4), reflecting ventricular depolarization along the direction of the precordial leads (Figure 1B). The Supporting Information Materials contain a more detailed description of the time-frequency averaging and depolarization map construction technique (Figures S1 and S2).

\subsection{Numerical parameters}

Two numerical parameters were computed: ventricular e-DYS and single lead local activation duration ( $\mathrm{x} x \mathrm{~d})$.

e-DYS was calculated as the maximal time difference between local activation times of leads V1-V8, using the center of mass of the signals (Figure 1B, right panel). This calculation is based on the idea that the UHF-QRS signal originates in depolarization wavefront ${ }^{9}$ and its morphology is the sum of stronger depolarization signals from adjacent areas to the electrode and weaker signals from remote areas. To predominantly analyze the nearest sources (maximal amplitudes), signals above 50\% threshold of baseline, relative to peak magnitude, were used. Threshold $50 \%$ was chosen after testing different levels between $10 \%$ and $60 \%$. Threshold $50 \%$ showed the highest stability and ability to distinguish between different types of pathologies and localization of slow conduction areas.

Vxd is defined as the duration of the UHF-QRS complex at half the maximum peak magnitude of ECG lead $x$ (Figure 1B, right panel). In this way, we assume that $\mathrm{Vxd}$ indicates the velocity of conduction underneath a certain electrode, low Vxd values pointing to high apparent conduction velocities, presumably due to the contribution of the intrinsic conduction system and high $V x d$ values to a nonhomogeneous substrate and presence of a scar. $V d$ is the mean of all $\mathrm{Vxd}$ values and measures the average depolarization propagation duration.

\section{RESULTS}

Figure 2 presents examples of LBBB patients with BiV and Hisbundle pacing. The depolarization activation maps show a high e-DYS and different local activation duration with the largest value occurring near lead V3 (indicated by V3d in the middle panel). BiV pacing clearly reduced the dyssynchrony, but an even stronger effect

\section{ventricular depolarization maps local activation duration}

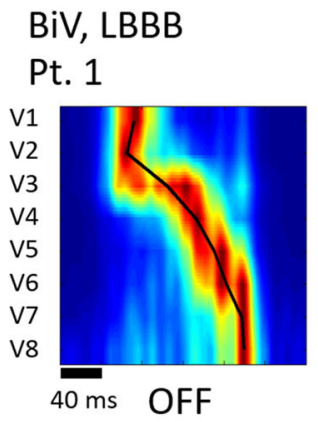

His, LBBB

Pt. 10
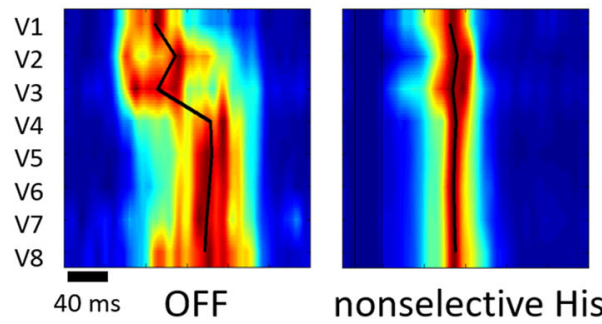

nonselective His

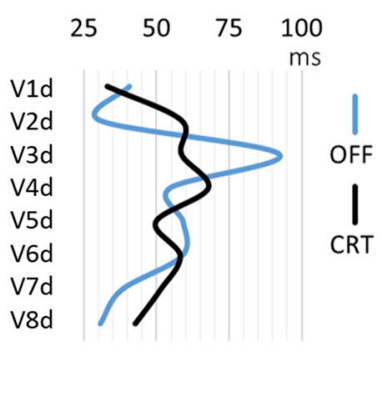

\begin{tabular}{c|ccc|}
\multicolumn{1}{c}{$\begin{array}{c}\text { e-DYS } \\
\text { ms }\end{array}$} & $\begin{array}{c}\text { Vd } \\
\text { ms }\end{array}$ & $\begin{array}{c}\text { QRSd } \\
\text { ms }\end{array}$ \\
\cline { 2 - 4 } OFF & 113 & 51 & 184 \\
BiV & 31 & 53 & 168 \\
\cline { 4 - 5 } & & &
\end{tabular}

$25 \quad 50 \quad 75100125$

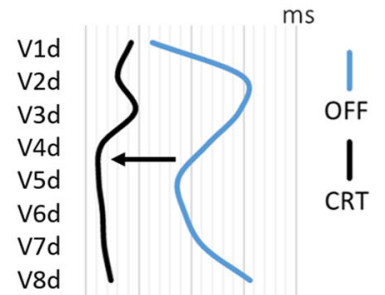

\begin{tabular}{|c|c|c|c|}
\hline & $\begin{array}{c}\text { e-DYS } \\
\mathrm{ms}\end{array}$ & $\begin{array}{l}\mathrm{Vd} \\
\mathrm{ms}\end{array}$ & $\begin{array}{c}\text { QRSd } \\
\mathrm{ms}\end{array}$ \\
\hline & 56 & 83 & 174 \\
\hline & 7 & 38 & 150 \\
\hline
\end{tabular}

depolarization propagation speed up, OFF vs. His-CRT

FIGURE 2 Upper panel: LBBB patient 1 before and during BiV. BiV significantly reduces e-DYS, whereas the Vd parameter (depolarization duration) slightly worsens. Bottom panel: LBBB patient 10 with heart failure, severe dysfunction of the left ventricle, dual chamber pacing mode, 60/minute, Atrioventricular delay 150/200 ms. BiV, biventricular resynchronization; e-DYS, electrical dyssynchrony 
was observed in the patient with His-bundle pacing. Both pacing techniques reduced QRS duration only modestly, whereas e-DYS was reduced to less than $30 \%$ of the baseline value. Moreover, activation duration $\mathrm{Vd}$ was hardly affected by BiV pacing, whereas it was strongly reduced by His-bundle pacing. The changes of e-DYS, Vd, and QRS duration during $\mathrm{BiV}$ and His-bundle pacing, relative to baseline, across all patients (Table 1) are shown in Figure 3. Nonselective His-bundle pacing significantly decreased both e-DYS and $\mathrm{Vd}$ while BiV only decreased e-DYS significantly. QRS duration did not show any significant change during either His-bundle or biventricular pacing.

Figure 4 shows the change in activation wavefront during progressive LV preexcitation, accompanied by a continuing reduction in e-DYS, but a minor change in Vd. Besides the strong visual impression of "straightening the wavefront," e-DYS seems to be more sensitive to $\mathrm{V}$ delay optimization than QRS duration.

Figure 5 shows results obtained in RBBB patients, resynchronized by BiV and His-bundle pacing. All three patients showed later activation in at least one of the leads V1, V2, and V3, supporting the diagnosis of RBBB. His-bundle pacing (patient 8, upper panels) and BiV pacing (patient 5, middle panel), created a more synchronous activation map, which was accompanied with a mild reduction in QRS duration but strong reductions in e-DYS and hardly any change in $\mathrm{Vd}$. In patient 6 (RBBB + left anterior hemiblock, lower panels) BiV pacing resulted in more asynchronous activation with now delays in leads $\mathrm{V} 7$ and $\mathrm{V} 8$, accompanied by an approximately $15 \%$ increase in QRS duration, but even more by approximately $30 \%$ increases in e-DYS and Vd, clearly demonstrating a detrimental effect of BiV pacing on conduction.

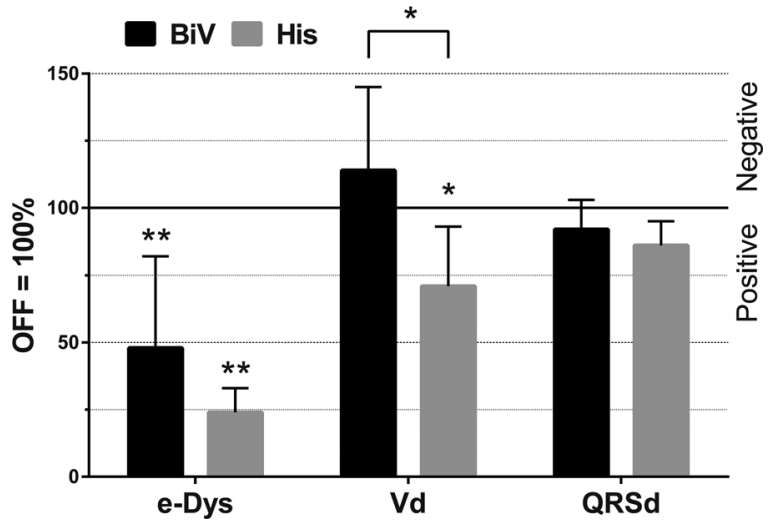

FIGURE 3 Baseline relative changes of mean e-DYS, Vd, and QRS duration (QRSd) between CRT OFF (100\%), BiV (mean from Pts 1-6, 10, 11), and nonselective His-bundle pacing (mean from Pts 7-11). Presented are mean values and standard deviations. A significant baseline relative decrease can be observed in e-DYS $(P<.01$, the Wilcoxon signed-rank test). e-DYS decreased more than QRS duration, during both biventricular pacing $(-50 \%$ vs $-8 \%)$ and His-bundle pacing $(-77 \%$ vs $-13 \%)$. A significant decrease can also be observed in $\mathrm{Vd}$ during nonselective His-bundle pacing $(P<.05)$. While biventricular pacing slightly increased $\mathrm{Vd}$, His-bundle pacing reduced $\mathrm{Vd}(+11 \%$ vs $-36 \%)$. A significant difference between biventricular and His-bundle pacing is in the Vd parameter ( ${ }^{*} P<.05$, ANOVA test, analysis of variance). This difference indicates the contribution of the fast conduction system. ${ }^{*} P<.05,{ }^{* *} P<.01$. ANOVA, analysis of variance; BiV, biventricular resynchronization; e-DYS, electrical dyssynchrony

The Supporting Information Materials contain a detailed description of all patients, complete numerical results, depolarization maps and a 12-lead ECG of all records before and during BiV and Hisbundle pacing.
BiV, LBBB

Pt. 4

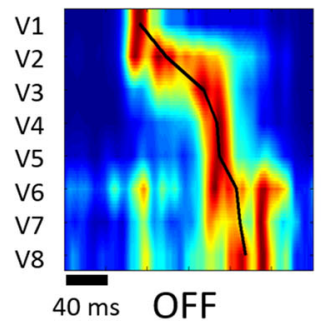

ventricular depolarization maps

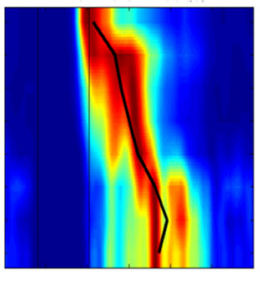

VV $0 \mathrm{~ms}$

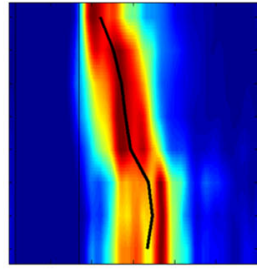

VV -20 ms

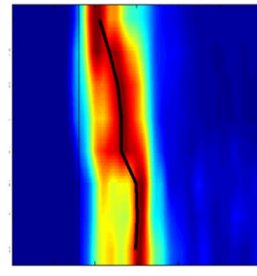

$\mathrm{VV}-40 \mathrm{~ms}$ local activation duration

\begin{tabular}{llllll|}
0 & 25 & 50 & 75 & 100 & 125
\end{tabular}

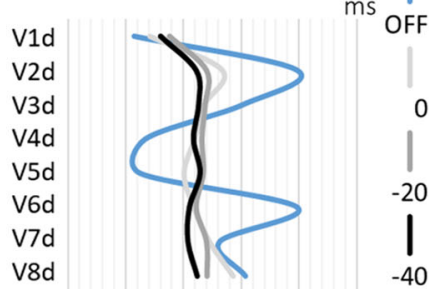

progressive LV pre-excitation

CRT OFF $=100 \%$

\begin{tabular}{l|ccc|}
\multicolumn{1}{c}{} & \multicolumn{1}{c}{$\begin{array}{c}\text { e-DYS } \\
\text { ms }\end{array}$} & $\begin{array}{c}\text { Vd } \\
\mathrm{ms}\end{array}$ & \multicolumn{1}{c}{$\begin{array}{c}\text { QRSd } \\
\mathrm{ms}\end{array}$} \\
\cline { 2 - 4 } \multicolumn{1}{l|}{ OFF } & 102 & 64 & 182 \\
VV 0 & 71 & 57 & 172 \\
VV -20 & 50 & 57 & 140 \\
VV -40 & 36 & 53 & 140 \\
\cline { 2 - 4 } N
\end{tabular}

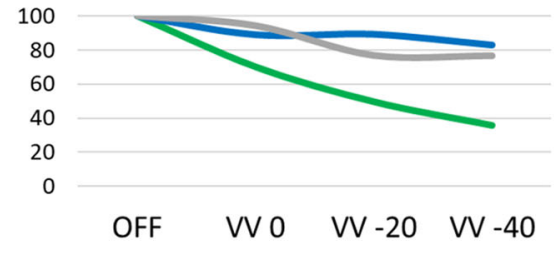

-e-DYS $-V d-$ QRSd

FIGURE 4 Biventricular pacing of LBBB patient 4. Progressive LV preexcitation: BiV OFF, BiV ON, VV delay = $0 \mathrm{~ms}$, VV delay $=-20 \mathrm{~ms}$, and $\mathrm{VV}$ delay $=-40 \mathrm{~ms}$. BiV, biventricular resynchronization; LBBB, Left bundle branch block 


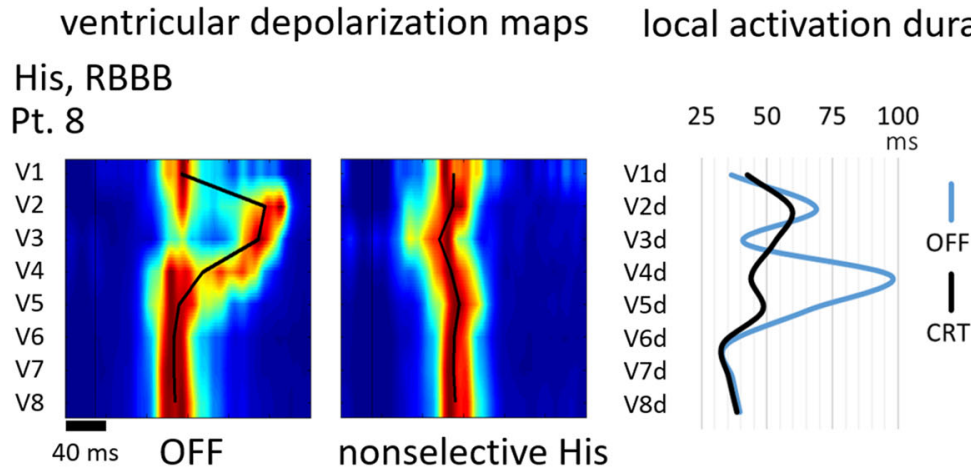

\begin{tabular}{lccc}
\multicolumn{1}{c}{$\begin{array}{c}\text { e-DYS } \\
\text { ms }\end{array}$} & \multicolumn{1}{c}{$\begin{array}{c}\text { Vd } \\
\text { ms }\end{array}$} & \multicolumn{1}{c}{$\begin{array}{c}\text { QRSd } \\
\text { ms }\end{array}$} \\
\cline { 2 - 4 } OFF & 89 & 53 & 172 \\
His & 24 & 44 & 160 \\
\cline { 2 - 4 } & & &
\end{tabular}
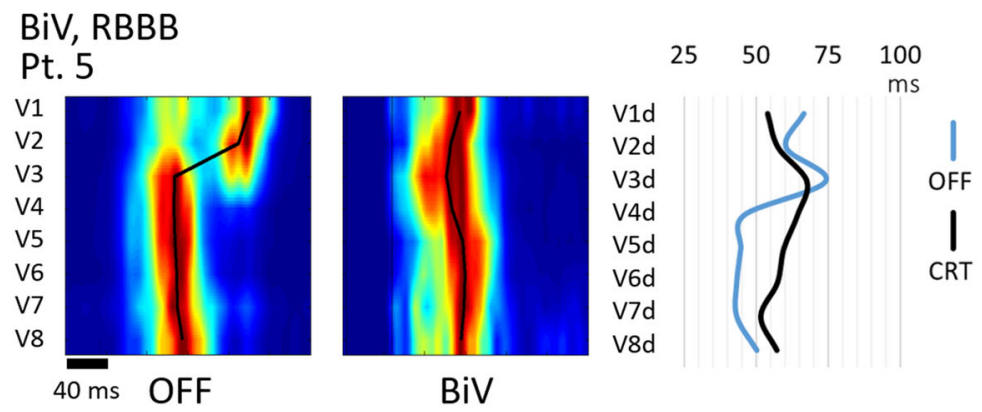

\begin{tabular}{c|ccc}
\multicolumn{1}{c}{$\begin{array}{c}\text { e-DYS } \\
\text { ms }\end{array}$} & $\begin{array}{c}\text { Vd } \\
\text { ms }\end{array}$ & \multicolumn{1}{c}{$\begin{array}{c}\text { QRSd } \\
\text { ms }\end{array}$} \\
\cline { 2 - 4 } OFF & 73 & 54 & 195 \\
BiV & 18 & 59 & 172 \\
\cline { 2 - 4 } & &
\end{tabular}

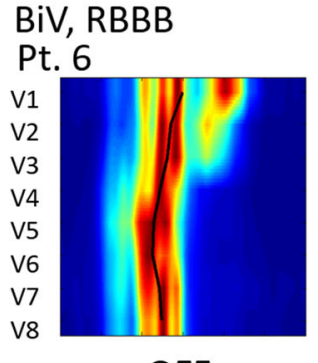

OFF

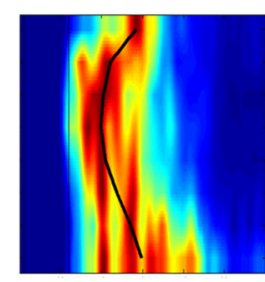

BiV
25

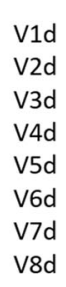

v8d

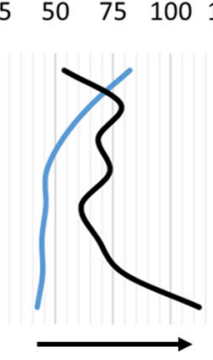

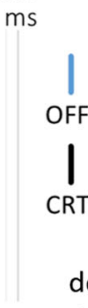

depolarization propagation deceleration OFF vs. BiV

FIGURE 5 Upper panel: RBBB patient 8 with a trifascicular block (Atrioventricular block of the 1st degree, RBBB + left anterior hemiblock) and syncope. Nonselective His-bundle pacing eliminates electrical dyssynchrony and speedup depolarization propagation. Middle panel: RBBB patient, BiV effectively eliminates electrical dyssynchrony, but myocardial pacing slightly decreases depolarization propagation speed. Bottom panel: RBBB + left anterior hemiblock patient, BiV increases dyssynchrony and slows down the depolarization propagation velocity.

$\mathrm{BiV}$, biventricular resynchronization; RBBB, right bundle branch block

\section{DISCUSSION}

The present study provides evidence that UHF-ECG provides more extensive and novel information about the nature of the conduction abnormality and of the achieved resynchronization in candidates for CRT. Importantly, the Vd, calculated from UHF-ECG, distinguishes between $\mathrm{BiV}$ and His-bundle pacing, suggesting a significant contribution of the fast conduction system whereas in cases with the scar, Vd was increased in leads near the scar. UHF-ECG also appears to provide valuable information with regard to the potential beneficial and harmful effects of CRT in RBBB patients.

\section{1 | Comparison of e-DYS, Vd, and QRS duration}

All data presented in this study demonstrate that the novel indices e-DYS, Vxd, and Vd provide a better and clearer picture of ventricular conduction than QRS duration. The difference between QRS duration on the one hand and e-DYS and $\mathrm{Vd}$, on the other hand, maybe explained by the fact that QRS duration is measured as the time between the earliest and latest activation of the ventricles, whereas the very early and late regions are not taken into account in e-DYS and $\mathrm{Vd}$, and presumably also hardly influence pump function, as these regions are small. Moreover, QRS duration alone cannot differentiate between the effects of dyssynchrony and tissue conduction velocity.

All data presented support the idea that e-DYS is more sensitive and still a robust parameter to express the degree of dyssynchrony. Its robustness stems from the fact that e-DYS is calculated from local activation times, estimated as the center of mass of depolarization activation distribution. The robustness is also achieved by using the large range of frequencies and the deterministic calculation.

Vxd provides completely novel information that is only obtained indirectly from other, invasive techniques. While the reduction in $\mathrm{Vxd}$ 
at the lead with the largest baseline Vxd shows that the slow conduction near that region is corrected by CRT, Vxd values of all leads, summarized in $\mathrm{Vd}$, indicates to what extent apparent tissue conduction is influenced. Because the location of pacing on the ventricle likely does not positively influence the conduction velocity, changes in Vd point toward the contribution of the rapid conduction system, such as in His-bundle pacing. Direct His-bundle stimulation activates the ventricles mainly through the His-Purkinje system, which is physiological and provides much faster ventricular depolarization. ${ }^{12,13}$ UHF-ECG recordings during His-bundle pacing show both a reduction in dyssynchrony and faster depolarization propagation in LBBB and RBBB patients, providing a possible explanation of why His-bundle pacing may provide better results than BiV pacing. ${ }^{14}$ Because pacing strategies such as His-bundle pacing and left bundle branch area pacing ${ }^{15}$ are rapidly gaining interest, $\mathrm{Vd}$ may be valuable in recognizing which patients benefit from these novel CRT approaches.

CRT can cause both positive and negative resynchronization effects in RBBB. ${ }^{16}$ Thus, RBBB patients may be another category of patients for whom UHF-ECG may be particularly of interest Especially in patients with RBBB and His-bundle pacing, UHF-ECG could be able to distinguish between the different types of ventricular capture. These are mainly nonselective His-bundle capture with or without BBB correction or pure myocardial paraHisian capture (Figure S6 Pt.8, R2, S7 Pt9, R2).

\subsection{A single depolarization map is more informative than a set of parameters}

While parameters expressing particular properties of electrical conduction are useful, anatomically oriented maps provide more in-depth information. Electrical mapping can be performed noninvasively by techniques like ECG imaging, ${ }^{17}$ but these measurements require a large number of electrodes and often a computerized totmography scan to determine the geometry of heart and chest. UHF-ECG provides a simpler and yet comprehensive picture of ventricular activation. In the present study, the overall morphology of the V1 to V8 ventricular depolarization maps show important features, such as the approximate location of the block in LBBB and RBBB and straightening of the activation wave, such as in VV optimization of BiV pacing. While the amount of information is undoubtedly less than obtained by ECG imaging, the great advantages of UHF-ECG are that it uses regular ECG with equipment that may be only slightly more expensive than regular equipment and directly fits in the current clinical workflow.

The activation maps show also features that are not expressed by $\mathrm{Vd}$ and e-DYS, such as slow or double activation (Figure 5 , patient 6, CRT OFF, lead V1). Double activation can cause artificial jumps in the calculated e-DYS value. Additionally, a double activation can carry information about different depolarization timing of the septum and RV free wall or about areas with slow depolarization propagation.

\subsection{Technical and data acquisition aspects}

The determination of accurate e-DYS requires meeting important ECG recording conditions. Averaging in time and frequency dimensions provide smooth and pronounced depolarization maps. Using lower quality 12-lead Holter records we have recently shown that $V_{E D}^{7}$ provides accurate identification of CRT responders in a large patient cohort. The technology used in the present study provides a better signal to noise ratio, and larger frequency range results in smaller variability in the depolarization maps and greater accuracy in the determination of e-DYS in particular patient. In addition, $\mathrm{Vd}$ is a new parameter, and its accurate determination is entirely conditioned by the broad-band ECG recording.

Although the UHF-ECG technology is slightly more complex, it uses the same electrodes as used for standard ECG analysis. Therefore, UHF-ECG may be swiftly applied in standardly used clinical settings. This may include measurements during a procedure of implantation or optimization, as the entire real-time recording and analysis take only 30 seconds. At present, higher sampling rates do not significantly increase the cost of the acquisition module in the ECG device.

UHF-ECG technology is currently being tested in five hospitals in the Czech Republic, Poland, and England. UHF-ECG is used for assessing ventricular electrical activation before pacemaker implantation and also during the postimplantation period for optimization of pacemaker settings. In one center, the UHF-ECG is used for the optimization of pacing electrode positioning during pacemaker implantation. To this purpose, the only condition required is a minimum length of UHF-ECG recording. This length of UHF-ECG recording may vary depending on the environmental conditions (artificial noise, more QRS morphologies within a single record). Practically we use recording intervals of 30 to 120 seconds.

\section{4 | Limitations}

So far, the concept behind e-DYS and Vd has not been validated by invasive electrophysiological measurements in either animals or patients. The interpretations of these parameters provided above are; however, supported by the various pacing interventions discussed in the results. Moreover, because e-DYS is largely determined by time differences between precordial leads near the LV and RV, it most likely expresses features similar to that as RV-LV e-DYS, defined as electrical synchronization index Esyn ${ }^{18}$ and ventricular electrical uncoupling VEU. ${ }^{19}$ Like e-DYS, Esyn, and VEU variables were shown to be more sensitive to CRT than QRS duration.

This study was designed to show the principle of the use of UHF-ECG to provide a better assessment of ventricular electrical activation. Patients were selected to demonstrate the potential of the new technology and its information content. Therefore, the study did not include the measurement of mechanical dyssynchrony and 
did not include a follow-up of the patients. The small size precludes statistical from a large cohort of patients.

\section{5 | CONCLUSIONS}

A new methodology for ventricular e-DYS, local depolarization duration $(\mathrm{V} x \mathrm{~d})$, and ventricular depolarization map computation from 14-lead (12-lead) ECG records have been introduced and discussed with examples. We show that UHF-ECG provides unique information about the ventricular electrical activation properties needed for targeted patient-optimized CRT, using basically regular ECG measurements albeit using higher frequencies.

\section{ACKNOWLEDGEMENTS}

The research was supported by Czech Science Foundation, project GA17-13830S; by Ministry of Education, Youth, and Sports of the Czech Republic, project L01212 and LQ1605; and Charles University Research Program Q38 and Research Centre program No. UNCE/ MED/002.

\section{ORCID}

Pavel Jurak (D) http://orcid.org/0000-0002-3793-5075 Karol Curila (D) http://orcid.org/0000-0003-3523-6358 Josef Halamek (iD http://orcid.org/0000-0001-8986-796X

\section{REFERENCES}

1. Asirvatham SJ. Cardiac resynchronization: is electrical synchrony relevant? J Cardiovasc Electrophysiol. 2007;18(10):1028-1031 https:// doi.org/10.1111/j.1540-8167.2007.00932.x

2. Van Deursen CJM, Blaauw $\mathrm{Y}$, Witjens $\mathrm{Ml}$, et al. The value of the 12-lead ECG for evaluation and optimization of cardiac resynchronization therapy in daily clinical practice. J Electrocardiol. 2014;47(2):202211. https://doi.org/10.1016/j.jelectrocard.2014.01.007

3. Langner PH, Geselowitz DB, Mansure FT, Lauer JA. High-frequency components in the electrocardiograms of normal subjects and of patients with coronary heart disease. Am Heart J. 1961;62:746-755.

4. Goldberger AL, Bhargava V, Froelicher V, et al. Effect of myocardial infarction on high-frequency QRS potentials. Circulation. 1981;64(1): 34-42.

5. Nichols TL, Mirvis DM. Frequency content of the electrocardiogram. Spatial features and effects of myocardial infarction. J Electrocardiol. 1985;18(2):185-194.

6. Moss AJ, Hall WJ, Cannom DS, et al. MADIT-CRT trial investigators. Cardiac-resynchronization therapy for the prevention of heart-failure events. N Engl J Med. 2009;361:1329-1338. https://doi.org/10.1056/ NEJMoa0906431

7. Plesinger F, Jurak P, Halamek J, et al. Ventricular electrical delay measured from body surface ECGs is associated with cardiac resynchronization therapy response in left bundle branch block patients from the MADIT-CRT trial (multicenter automatic defibrillator implantation-cardiac resynchronization therapy response in left bundle branch block patients from the MADIT-CRT trial (multicenter automatic defibrillator implantation-cardiac resynchronization therapy). Circ Arrhythmia Electrophysiol. 2018;11(5):e005719. https://doi. org/10.1161/circep.117.005719

8. Halamek J, Leinveber P, Viscor I, et al. The relationship between ECG predictors of cardiac resynchronization therapy benefit. PLoS One. 2019; 14(5):1-10. https://doi.org/10.1371/journal.pone.0217097

9. Jurak P, Halamek J, Meluzin J, et al. Ventricular dyssynchrony assessment using ultra-high frequency ECG technique. J Interv Card Electrophysiol. 2017;49(3):245-254. https://doi.org/10.1007/s10840-017-0268-0

10. Andrla $P$, Plesinger $F$, Halamek J, Leinveber P, Viscor I, Jurak P. A method for removing pacing artifacts from ultra-high-frequency electrocardiograms. 2018 Comput Cardiol Conf. 2018;45:1-5. https:// doi.org/10.22489/cinc.2018.106

11. Plesinger F, Jurco J, Jurak P. Robust multichannel QRS detection. Comput Cardiol. 2014;2014:557-560.

12. Arnold AD, Shun-Shin MJ, Keene D, et al. His resynchronization versus biventricular pacing in patients with heart failure and left bundle branch block. J Am Coll Cardiol. 2018;72(24):3112-3122. https://doi.org/10.1016/j.jacc.2018.09.073

13. Sharma PS, Dandamudi G, Herweg B, et al. Permanent His-bundle pacing as an alternative to biventricular pacing for cardiac resynchronization therapy: a multicenter experience. Heart Rhythm. 2018;15(3):413-420. https://doi.org/10.1016/j.hrthm.2017. 10.014

14. Upadhyay GA, Vijayaraman P, Nayak HM, et al. On-treatment comparison between corrective His bundle pacing and biventricular pacing for cardiac resynchronization: a secondary analysis of HisSYNC. Heart Rhythm. 2019;16:1797-1807. https://doi.org/10.1016/j. hrthm.2019.05.009

15. Vijayaraman P, Huang W. Atrioventricular block at the distal His bundle: electrophysiological insights from left bundle branch pacing. Heart Rhythm Case Reports. 2019;5:233-236J. https://doi.org/10. 1016/j.hrcr.2019.01.006

16. Auricchio A, Lumens J, Prinzen FW. Cardiac resynchronization therapy has a role in patients with right bundle branch block. Circ Arrhythmia Electrophysiol. 2014;7(3):532-541. https://doi.org/10. 1161/CIRCEP.113.000628

17. Ploux S, Lumens J, Whinnett Z, et al. Noninvasive electrocardiographic mapping to improve patient selection for cardiac resynchronization therapy. J Am Coll Cardiol. 2013;61(24):2435-43.

18. Jia P, Ramanathan C, Ghanem RN, Ryu K, Varma N, Rudy Y. Electrocardiographic imaging of cardiac resynchronization therapy in heart failure: observation of variable electrophysiologic responses. Heart Rhythm. 2006;3(3):296-310. https://doi.org/10.1016/j.hrthm.2005.11.025

19. Huntjens $P$, Ploux $S$, Strik $M$, et al. Electrical substrates driving response to cardiac resynchronization therapy: a combined clinical-computational evaluation. Circ Arrhythm Electrophysiol. 2018;11(4):1-12. https://doi.org/ 10.1161/CIRCEP.118.006613

\section{SUPPORTING INFORMATION}

Additional supporting information may be found online in the Supporting Information section.

How to cite this article: Jurak $\mathrm{P}$, Curila $\mathrm{K}$, Leinveber $\mathrm{P}$, et al. Novel ultra-high-frequency electrocardiogram tool for the description of the ventricular depolarization pattern before and during cardiac resynchronization. J Cardiovasc Electrophysiol. 2020;31:300-307. https://doi.org/10.1111/jce.14299 